\title{
A Novel Optimization Method for Nonconvex Quadratically Constrained Quadratic Programs
}

\author{
Hongwei Jiao, ${ }^{1}$ Yong-Qiang Chen, ${ }^{2}$ and Wei-Xin Cheng ${ }^{2}$ \\ ${ }^{1}$ School of Mathematical Science, Henan Institute of Science and Technology, Xinxiang 453003, China \\ ${ }^{2}$ College of Mathematics and Information Science, Henan Normal University, Xinxiang 453007, China \\ Correspondence should be addressed to Hongwei Jiao; jhwxd2014@126.com and Wei-Xin Cheng; chengweixin2014@126.com
}

Received 21 February 2014; Revised 26 March 2014; Accepted 26 March 2014; Published 27 April 2014

Academic Editor: Yisheng Song

Copyright (c) 2014 Hongwei Jiao et al. This is an open access article distributed under the Creative Commons Attribution License, which permits unrestricted use, distribution, and reproduction in any medium, provided the original work is properly cited.

\begin{abstract}
This paper presents a novel optimization method for effectively solving nonconvex quadratically constrained quadratic programs (NQCQP) problem. By applying a novel parametric linearizing approach, the initial NQCQP problem and its subproblems can be transformed into a sequence of parametric linear programs relaxation problems. To enhance the computational efficiency of the presented algorithm, a cutting down approach is combined in the branch and bound algorithm. By computing a series of parametric linear programs problems, the presented algorithm converges to the global optimum point of the NQCQP problem. At last, numerical experiments demonstrate the performance and computational superiority of the presented algorithm.
\end{abstract}

\section{Introduction}

The nonconvex quadratically constrained quadratic programs problems have attracted the attention of practitioners and researchers for 30 years. During the past 10 years, curiosity in these problems has been especially intense. In part, this is because the NQCQP problems have a large number of practical applications, for example, pooling problems in petrochemistry [1], modularization of product subassemblies [2], chance-constrained optimization problems, production planning or portfolio optimization [3-5], the fuel mixture problem encountered in oil industry [6], and also placement and layout problems appeared in integrated circuit design (see $[7,8]$ ). In addition, many nonlinear optimization problems can be transformed into the form, for example, special classes of structured stochastic games [9] can be interpreted as quadratic programs problems, the packing problem contained in the unit square can be formulated as concave quadratic constraints quadratic programs problem, $\{0,1\}$ variable in $0-1$ programming may be also represented by concave quadratic constraints, and minmax location problems [4] also lead to quadratic programs problems with quadratic constraints. Another cause for the strong attention in the NQCQP problems is that, from a research point of view, the class of problems put forward significant theoretical and computational defiance. This is mainly because these problems are global optimization problem; that is, they are well known to generally own multiple local optimum points that are not globally optimum point. Therefore, it is very essential to put forward a good global optimization method for solving the NQCQP problems.

In this paper, we will investigate the following NQCQP problems:

$$
\begin{array}{lr}
\min \quad G_{0}(x)=x^{T} A^{0} x+\left(d^{0}\right)^{T} x \\
\text { s.t. } \quad G_{i}(x)=x^{T} A^{i} x+\left(d^{i}\right)^{T} x \leq b_{i}, \\
i=1, \ldots, m, \\
\\
x \in X^{0}=\left\{x \in R^{n}: l^{0} \leq x \leq u^{0}\right\},
\end{array}
$$

where $A^{i}=\left(a_{j k}^{i}\right)_{n \times n} \in R^{n \times n}(i=0,1, \ldots, m)$ are all symmetric matrices, $d^{0}, d^{i} \in R^{n}, b_{i} \in R, i=1, \ldots, m$, and $l^{0}=$ $\left(l_{1}^{0}, \ldots, l_{n}^{0}\right)^{T}, u^{0}=\left(u_{1}^{0}, \ldots, u_{n}^{0}\right)^{T}$.

In last several decades, some algorithms have been exploited for globally solving the special case or general case of the NQCQP problems. For example, based on a 
novel reformulation-linearization/convexification approach, Sherali and Tuncbilek [10] proposed a global optimization algorithm for linearly constrained nonconvex quadratic programs problems. Based on outer approximation and branch and bound scheme by solving linear programs subproblems, Al-Khayyal et al. [7] presented an algorithm for computing the approximate global optimal solutions of the NQCQP problems. Based on Lagrangian underestimation method to compute lower bounds and utilize the Interval Newton method to facilitate the convergence of algorithm in the neighborhood of the global optimum point, Van Voorhis [11] developed a branch and bound algorithm for globally solving the NQCQP problems. By partitioning the feasible region into the Cartesian product of two-dimensional triangles and rectangles and by utilizing the convex and concave envelopes of bilinear functions over triangles and rectangles, a simplicial branch and bound algorithm [12] was presented for solving globally the NQCQP problems. Based on semidefinite relaxations and finite KKT-branching method, Burer and Vandenbussche [13] presented a finite branch and bound algorithm for globally solving the NQCQP problems. In [14], Zheng et al. presented a decomposition-approximation method for constructing convex relaxations of the NQCQP problems, which can be used to offer a tighter lower bound for solving the problems (NQCQP). Using duality bounds approach, Thoai [15] presented a branch and bound algorithm for solving the NQCQP problems. Based on linear relaxation approximation technique and linearity-based range deleting tactics, Gao et al. [16] presented a rectangle branch and reduce method for the linearly constrained quadratic programs problems; by utilizing linearizing technique and quadratic constraint-based range compressing technique, Gao et al. [17] presented a branch and reduce approach for globally solving the NQCQP problems. By utilizing the special structure of quadratic function and linearization technique, Shen et al. [18] and Shen and Liu [19] proposed two effective global optimization algorithms for computing the NQCQP problems. By making use of linear relaxation approximation technique, Qu et al. [20] and Jiao and Chen [21] proposed two deterministic algorithms for calculating the NQCQP problems. Except for the above reviewed references, several algorithms for solving generalized geometric programming problem presented in [22-30] can be also used to solve the NQCQP problems. For an excellent review of recent advances in global optimization, the reader is referred to Floudas and Gounaris [31].

In this paper, by combing a parametric linearizing approach with a cutting down approach, we will present a novel global optimization method for solving the NQCQP problems. The main characteristics of the presented approach are expounded as follows. Firstly, a novel linear relaxation approximation technique, that is, a parametric linearizing approach, is constructed for inconsistently transforming the NQCQP problems into a sequence of parametric linear programs relaxation problems, and by subsequently subdividing the initial hyperrectangle the optimal point of parametric linear programs relaxation problems can infinitely approach the global optimum point of the problem (NQCQP). Secondly, the proposed parametric linear programs relaxation problems are putted into a branch and bound framework without adding any new variables and constraint functions and which can be easily calculated by any effective linear programs algorithm procedure. Thirdly, a cutting down approach is exploited to eliminate a large part of the currently investigated subhyperrectangle which does not contain the global optimum point of the problem (NQCQP). Combing the parametric linear programs relaxation problem with the cutting down approach in a branch and bound procedure, a new optimization method is displayed for globally solving the NQCQP problems. At last, numerical results indicate that the proposed method can be employed to obtain the global optimum point of the problem (NQCQP).

The remainder of this paper is composed as follows. The next section describes a novel parametric linearizing approach and the parametric linear programs relaxation of the problem (NQCQP) is constructed. In Section 3 a cutting down approach is presented. Section 4 combines the cutting down approach within a branch and bound scheme; an optimization algorithm and its global convergence are described. In Section 5 some test examples and their results are reported to demonstrate the feasibility and superiority of the presented algorithm. At last, some concluding remarks are described.

\section{Parametric Linear Relaxation}

The principal composition in the configuration of a branch and bound algorithm for globally solving the problem (NQCQP) is the computation of lower bounds of the problem (NQCQP) and its divided subproblems. The lower bounds of the global minimum values of the problem (NQCQP) and its divided subproblems can be calculated by solving a series of parametric linear programs relaxation problem (PLPRP). In order to construct the PLPRP, the proposed approach is to replace each quadratic function $G_{i}(x)$ by a parametric linear function.

Let $X=\left(X_{j}\right)_{n \times 1} \subseteq X^{0}$ with $X_{j}=\left[l_{j}, u_{j}\right](j=1, \ldots, n)$. For each $i=0,1, \ldots, m$, define $x\left(\theta_{i}\right)=l+\theta_{i}(u-l)$, where $\theta_{i} \in$ $\{0,1\}, l^{T}=\left\{l_{1}, l_{2}, \ldots, l_{n}\right\}, u^{T}=\left\{u_{1}, u_{2}, \ldots, u_{n}\right\}, x(0)=l$, and $x(1)=u$. For convenience in expression, for all $x \in X \subseteq X^{0}$, for each $i=0,1, \ldots, m$, some symbols are given as follows:

$$
\underline{Z}_{i}=2 \times\left(\begin{array}{c}
\sum_{k=1, a_{1 k}^{i}>0}^{n} a_{1 k}^{i} l_{k}+\sum_{k=1, a_{1 k}^{i}<0}^{n} a_{1 k}^{i} u_{k} \\
\sum_{k=1, a_{2 k}^{i}>0}^{n} a_{2 k}^{i} l_{k}+\sum_{k=1, a_{2 k}^{i}<0}^{n} a_{2 k}^{i} u_{k} \\
\vdots \\
\sum_{k=1, a_{n k}^{i}>0}^{n} a_{n k}^{i} l_{k}+\sum_{k=1, a_{n k}^{i}<0}^{n} a_{n k}^{i} u_{k}
\end{array}\right),
$$




$$
\begin{gathered}
\bar{Z}_{i}=2 \times\left(\begin{array}{c}
\sum_{k=1, a_{1 k}^{i}>0}^{n} a_{1 k}^{i} u_{k}+\sum_{k=1, a_{1 k}^{i}<0}^{n} a_{1 k}^{i} l_{k} \\
\sum_{k=1, a_{2 k}^{i}>0}^{n} a_{2 k}^{i} u_{k}+\sum_{k=1, a_{2 k}^{i}<0}^{n} a_{2 k}^{i} l_{k} \\
\vdots \\
\sum_{k=1, a_{n k}^{i}>0}^{n} a_{n k}^{i} u_{k}+\sum_{k=1, a_{n k}^{i}<0}^{n} a_{n k}^{i} l_{k}
\end{array}\right), \\
f_{i}(x)=x^{T} A^{i} x, \\
Z_{i}\left(\theta_{i}\right)=\underline{Z}_{i}+\theta_{i}\left(\bar{Z}_{i}-\underline{Z}_{i}\right), \\
Z_{i}\left(1-\theta_{i}\right)=\underline{Z}_{i}+\left(1-\theta_{i}\right)\left(\bar{Z}_{i}-\underline{Z}_{i}\right), \\
f_{i}^{l}\left(x, X, \theta_{i}\right)=Z_{i}\left(\theta_{i}\right)^{T} x+x\left(\theta_{i}\right)^{T} A^{i} x\left(\theta_{i}\right) \\
-Z_{i}\left(\theta_{i}\right)^{T} x\left(\theta_{i}\right), \\
f_{i}^{u}\left(x, X, \theta_{i}\right)=Z_{i}\left(1-\theta_{i}\right)^{T} x+x\left(\theta_{i}\right)^{T} A^{i} x\left(\theta_{i}\right) \\
-Z_{i}\left(1-\theta_{i}\right)^{T} x\left(\theta_{i}\right) .
\end{gathered}
$$

Obviously, we have

$$
Z_{i}(0)=\underline{Z}_{i}, \quad Z_{i}(1)=\bar{Z}_{i} .
$$

Theorem 1. For any $x \in X \subseteq X^{0}$, for each $i=0,1, \ldots, m$, consider the function $f_{i}(x), f_{i}^{l}\left(x, X, \theta_{i}\right)$ and $f_{i}^{u}\left(x, X, \theta_{i}\right)$; then the following conclusions hold:

(i) $f_{i}^{l}\left(x, X, \theta_{i}\right) \leq f_{i}(x) \leq f_{i}^{u}\left(x, X, \theta_{i}\right)$;

(ii) $\left\|f_{i}^{u}\left(x, X, \theta_{i}\right)-f_{i}(x)\right\| \rightarrow 0$ and $\| f_{i}(x)-$ $f_{i}^{l}\left(x, X, \theta_{i}\right) \| \rightarrow 0$ as $\|u-l\| \rightarrow 0$.

Proof. (i) The gradient function of the function $f_{i}(x)=$ $x^{T} A^{i} x, i=0,1, \ldots, m$, can be expressed as follows:

$$
\frac{\partial f_{i}(x)}{\partial x}=2 A^{i} x=2 \times\left(\begin{array}{c}
\sum_{k=1}^{n} a_{1 k}^{i} x_{k} \\
\sum_{k=1}^{n} a_{2 k}^{i} x_{k} \\
\vdots \\
\sum_{k=1}^{n} a_{n k}^{i} x_{k}
\end{array}\right) .
$$

Obviously, we have

$$
\underline{Z}_{i} \leq \frac{\partial f_{i}(x)}{\partial x}=2 A^{i} x \leq \bar{Z}_{i}
$$

By the mean value theorem, for any $x \in X$, there exists a point $\xi=\alpha x+(1-\alpha) x\left(\theta_{i}\right)$, where $\alpha \in[0,1]$, such that

$$
\begin{aligned}
f_{i}(x) & =f_{i}\left(x\left(\theta_{i}\right)\right)+\left(\frac{\partial f_{i}(\xi)}{\partial \xi}\right)^{T}\left(x-x\left(\theta_{i}\right)\right) \\
& =x\left(\theta_{i}\right)^{T} A^{i} x\left(\theta_{i}\right)+\left(\frac{\partial f_{i}(\xi)}{\partial \xi}\right)^{T}\left(x-x\left(\theta_{i}\right)\right) .
\end{aligned}
$$

If $\theta_{i}=0$, then we have

$$
\begin{gathered}
\frac{\partial f_{i}(\xi)}{\partial \xi} \geq \underline{Z}_{i}=Z_{i}\left(\theta_{i}\right), \\
x-x\left(\theta_{i}\right)=x-l \geq 0, \quad \text { for any } x \in X .
\end{gathered}
$$

If $\theta_{i}=1$, it follows that

$$
\begin{gathered}
\frac{\partial f_{i}(\xi)}{\partial \xi} \leq \bar{Z}_{i}=Z_{i}\left(\theta_{i}\right), \\
x-x\left(\theta_{i}\right)=x-u \leq 0, \quad \text { for any } x \in X .
\end{gathered}
$$

Therefore, we can get that

$$
\begin{aligned}
f_{i}(x) & =x\left(\theta_{i}\right)^{T} A^{i} x\left(\theta_{i}\right)+\left(\frac{\partial f_{i}(\xi)}{\partial \xi}\right)^{T}\left(x-x\left(\theta_{i}\right)\right) \\
& \geq x\left(\theta_{i}\right)^{T} A^{i} x\left(\theta_{i}\right)+Z_{i}\left(\theta_{i}\right)^{T}\left(x-x\left(\theta_{i}\right)\right) \\
& =f_{i}^{l}\left(x, X, \theta_{i}\right) .
\end{aligned}
$$

Analogously, if $\theta_{i}=0$, then it follows that

$$
\begin{gathered}
\frac{\partial f_{i}(\xi)}{\partial \xi} \leq \bar{Z}_{i}=Z_{i}\left(1-\theta_{i}\right), \\
x-x\left(\theta_{i}\right)=x-l \geq 0, \quad \text { for any } x \in X .
\end{gathered}
$$

If $\theta_{i}=1$, the following inequalities hold:

$$
\begin{gathered}
\frac{\partial f_{i}(\xi)}{\partial \xi} \geq \underline{Z}_{i}=Z_{i}\left(1-\theta_{i}\right), \\
x-x\left(\theta_{i}\right)=x-u \leq 0, \quad \text { for any } x \in X .
\end{gathered}
$$

Hence, it follows as above that

$$
\begin{aligned}
f_{i}(x) & =x\left(\theta_{i}\right)^{T} A^{i} x\left(\theta_{i}\right)+\left(\frac{\partial f_{i}(\xi)}{\partial \xi}\right)^{T}\left(x-x\left(\theta_{i}\right)\right) \\
& \leq x\left(\theta_{i}\right)^{T} A^{i} x\left(\theta_{i}\right)+Z_{i}\left(1-\theta_{i}\right)^{T}\left(x-x\left(\theta_{i}\right)\right) \\
& =f_{i}^{u}\left(x, X, \theta_{i}\right) .
\end{aligned}
$$

The conclusion (i) is followed. 
(ii) Consider $\left\|f_{i}(x)-f_{i}^{l}\left(x, X, \theta_{i}\right)\right\|$, we have

$$
\begin{aligned}
& \left\|f_{i}(x)-f_{i}^{l}\left(x, X, \theta_{i}\right)\right\| \\
= & \left\|x^{T} A^{i} x-\left[Z_{i}\left(\theta_{i}\right)^{T} x+x\left(\theta_{i}\right)^{T} A^{i} x\left(\theta_{i}\right)-Z_{i}\left(\theta_{i}\right)^{T} x\left(\theta_{i}\right)\right]\right\| \\
= & \|\left[x\left(\theta_{i}\right)^{T} A^{i} x\left(\theta_{i}\right)+\left(\frac{\partial f_{i}(\xi)}{\partial \xi}\right)^{T}\left(x-x\left(\theta_{i}\right)\right)\right] \\
= & \left\|\left(\frac{\partial f_{i}(\xi)}{\partial \xi}\right)^{T}\left(x-x\left(\theta_{i}\right)\right)-\left[Z_{i}\left(\theta_{i}\right)^{T} x-Z_{i}\left(\theta_{i}\right)^{T} x\left(\theta_{i}\right)\right]\right\| \\
= & \left\|\left(\frac{\partial f_{i}(\xi)}{\partial \xi}-Z_{i}\left(\theta_{i}\right)\right)^{T}\left(x-x\left(\theta_{i}\right)\right)\right\| \\
\leq & \left\|\frac{\partial f_{i}(\xi)}{\partial \xi}-Z_{i}\left(\theta_{i}\right)\right\|\left\|x-x\left(\theta_{i}\right)\right\| \\
\leq & \left\|\bar{Z}_{i}-\underline{Z}_{i}\right\|\left\|x-x\left(\theta_{i}\right)\right\| \\
\leq & \left\|\bar{Z}_{i}-\underline{Z}_{i}\right\|\|u-l\|,
\end{aligned}
$$

where $\xi=\alpha x+(1-\alpha) x\left(\theta_{i}\right)$ with $\alpha \in[0,1]$.

Since $\left\|\bar{Z}_{i}-\underline{Z}_{i}\right\|\|u-l\| \rightarrow 0$ as $\|u-l\| \rightarrow 0$, thus we can get that

$$
\left\|f_{i}(x)-f_{i}^{l}\left(x, X, \theta_{i}\right)\right\| \longrightarrow 0 \text { as }\|u-l\| \longrightarrow 0 .
$$

Similarly, we can prove that

$$
\left\|f_{i}^{u}\left(x, X, \theta_{i}\right)-f_{i}(x)\right\| \longrightarrow 0 \text { as }\|u-l\| \longrightarrow 0 .
$$

The conclusion is complete.

By Theorem 1 , for any $x \in X$, for each $i=0,1, \ldots, m$, we define

$$
\begin{aligned}
G_{i}^{L}\left(x, X, \theta_{i}\right)= & Z_{i}\left(\theta_{i}\right)^{T} x+x\left(\theta_{i}\right)^{T} A^{i} x\left(\theta_{i}\right) \\
& -Z_{i}\left(\theta_{i}\right)^{T} x\left(\theta_{i}\right)+\left(d^{i}\right)^{T} x, \\
G_{i}^{U}\left(x, X, \theta_{i}\right)= & Z_{i}\left(1-\theta_{i}\right)^{T} x+x\left(\theta_{i}\right)^{T} A^{i} x\left(\theta_{i}\right) \\
& -Z_{i}\left(1-\theta_{i}\right)^{T} x\left(\theta_{i}\right)+\left(d^{i}\right)^{T} x .
\end{aligned}
$$

Theorem 2. For any $x \in X \subseteq X^{0}$, for each $i=0,1, \ldots, m$, the following conclusions hold:

(i) $G_{i}^{L}\left(x, X, \theta_{i}\right) \leq G_{i}(x) \leq G_{i}^{U}\left(x, X, \theta_{i}\right)$;

(ii) $\left\|G_{i}^{U}\left(x, X, \theta_{i}\right)-G_{i}(x)\right\| \rightarrow 0$ and $\| G_{i}(x)-$ $G_{i}^{L}\left(x, X, \theta_{i}\right) \| \rightarrow 0$ as $\|u-l\| \rightarrow 0$.
Proof. (i) By the definitions of $G_{i}^{L}\left(x, X, \theta_{i}\right), G_{i}(x)$ and $G_{i}^{U}\left(x, X, \theta_{i}\right)$ and the conclusion (i) of Theorem 1, we have

$$
\begin{aligned}
G_{i}^{L} & \left(x, X, \theta_{i}\right) \\
& =Z_{i}\left(\theta_{i}\right)^{T} x+x\left(\theta_{i}\right)^{T} A^{i} x\left(\theta_{i}\right)-Z_{i}\left(\theta_{i}\right)^{T} x\left(\theta_{i}\right)+\left(d^{i}\right)^{T} x \\
& =f_{i}^{l}\left(x, X, \theta_{i}\right)+\left(d^{i}\right)^{T} x \\
\leq & f_{i}(x)+\left(d^{i}\right)^{T} x \\
= & G_{i}(x) \\
\leq & f_{i}^{u}\left(x, X, \theta_{i}\right)+\left(d^{i}\right)^{T} x \\
= & Z_{i}\left(1-\theta_{i}\right)^{T} x+x\left(\theta_{i}\right)^{T} A^{i} x\left(\theta_{i}\right) \\
& -Z_{i}\left(1-\theta_{i}\right)^{T} x\left(\theta_{i}\right)+\left(d^{i}\right)^{T} x \\
= & G_{i}^{U}\left(x, X, \theta_{i}\right) .
\end{aligned}
$$

Therefore, the conclusion (i) is followed.

(ii) By the expressions of $G_{i}^{L}\left(x, X, \theta_{i}\right), G_{i}(x), G_{i}^{U}\left(x, X, \theta_{i}\right)$, $f_{i}^{l}\left(x, X, \theta_{i}\right), f_{i}(x)$, and $f_{i}^{u}\left(x, X, \theta_{i}\right)$, we can get that

$$
\begin{array}{r}
\left\|G_{i}(x)-G_{i}^{L}\left(x, X, \theta_{i}\right)\right\| \\
=\|\left[x^{T} A^{i} x+\left(d^{i}\right)^{T} x\right]-\left[Z_{i}\left(\theta_{i}\right)^{T} x+x\left(\theta_{i}\right)^{T} A^{i} x\left(\theta_{i}\right)\right. \\
\left.-Z_{i}\left(\theta_{i}\right)^{T} x\left(\theta_{i}\right)+\left(d^{i}\right)^{T} x\right] \|
\end{array}
$$$$
=\left\|x^{T} A^{i} x-\left[Z_{i}\left(\theta_{i}\right)^{T} x+x\left(\theta_{i}\right)^{T} A^{i} x\left(\theta_{i}\right)-Z_{i}\left(\theta_{i}\right)^{T} x\left(\theta_{i}\right)\right]\right\|
$$$$
=\left\|f_{i}(x)-f_{i}^{l}\left(x, X, \theta_{i}\right)\right\|,
$$$$
\left\|G_{i}^{U}\left(x, X, \theta_{i}\right)-G_{i}(x)\right\|
$$$$
=\|\left[Z_{i}\left(1-\theta_{i}\right)^{T} x+x\left(\theta_{i}\right)^{T} A^{i} x\left(\theta_{i}\right)-Z_{i}\left(1-\theta_{i}\right)^{T} x\left(\theta_{i}\right)\right.
$$$$
\left.+\left(d^{i}\right)^{T} x\right]-\left[x^{T} A^{i} x+\left(d^{i}\right)^{T} x\right] \|
$$$$
=\|\left[Z_{i}\left(1-\theta_{i}\right)^{T} x+x\left(\theta_{i}\right)^{T} A^{i} x\left(\theta_{i}\right)\right.
$$

$$
\left.-Z_{i}\left(1-\theta_{i}\right)^{T} x\left(\theta_{i}\right)\right]-x^{T} A^{i} x \|
$$

$=\left\|f_{i}^{u}\left(x, X, \theta_{i}\right)-f_{i}(x)\right\|$. 
By the proof of Theorem 1 (ii), we can get

$$
\begin{aligned}
& \left\|G_{i}^{U}\left(x, X, \theta_{i}\right)-G_{i}(x)\right\| \\
& \quad=\left\|f_{i}^{u}\left(x, X, \theta_{i}\right)-f_{i}(x)\right\| \longrightarrow 0 \quad \text { as }\|u-l\| \longrightarrow 0, \\
& \left\|G_{i}(x)-G_{i}^{L}\left(x, X, \theta_{i}\right)\right\| \\
& \quad=\left\|f_{i}(x)-f_{i}^{l}\left(x, X, \theta_{i}\right)\right\| \longrightarrow 0 \text { as }\|u-l\| \longrightarrow 0 .
\end{aligned}
$$

The conclusion is complete.

By Theorem 2, we can construct the underestimating approximation parametric linear programs relaxation problem (PLPRP) of the problem (NQCQP) over subhyperrectangle $X$ as follows:

$$
\begin{array}{ll}
\min & G_{0}^{L}\left(x, X, \theta_{0}\right), \\
\text { s.t. } & G_{i}^{L}\left(x, X, \theta_{i}\right) \leq b_{i}, \quad i=1, \ldots, m, \\
& x \in X=\{x: l \leq x \leq u\} \subseteq X^{0},
\end{array}
$$

where

$$
\begin{aligned}
G_{i}^{L}\left(x, X, \theta_{i}\right)= & Z_{i}\left(\theta_{i}\right)^{T} x+x\left(\theta_{i}\right)^{T} A^{i} x\left(\theta_{i}\right) \\
& -Z_{i}\left(\theta_{i}\right)^{T} x\left(\theta_{i}\right)+\left(d^{i}\right)^{T} x .
\end{aligned}
$$

Based on the above parametric linearizing approach, every feasible point of the problem (NQCQP) is feasible to the problem (PLPRP) over the subhyperrectangle $X$, and the objective function value of the problem (PLPRP) at each feasible point is less than or equal to that of the problem (NQCQP) over the subhyperrectangle $X$. Thus, the optimal value of the problem (PLPRP) offers a valid lower bound for the optimal value of the problem (NQCQP) over the subhyperrectangle $X$.

\section{Cutting Down Approach}

To enhance the computational speed of the investigated algorithm, based on the above parametric linear relaxation problem, a novel cutting down approach is described in the following Theorem 3 . At the $k$-th iteration of the proposed algorithm, we will judge whether or not the subhyperrectangle $X$ contains a global optimum point of the problem (NQCQP). The cutting down approach can be used to reject a part of the subhyperrectangle $X$ or the whole $X$ without deleting any global optimum point of the problem (NQCQP). For convenience, for any $x \in X=\left(X_{j}\right)_{n \times 1}$ with $X_{j}=\left[l_{j}, u_{j}\right](j=1, \ldots, n)$, without loss of generality, we express the $G_{i}^{L}\left(x, X, \theta_{i}\right)$ in the problem (PLPRP) over the subhyperrectangle $X$ as the following form:

$$
G_{i}^{L}\left(x, X, \theta_{i}\right)=\sum_{j=1}^{n} \gamma_{i j}\left(\theta_{i}\right) x_{j}+\eta_{i}\left(\theta_{i}\right), \quad i=0,1, \ldots, m .
$$

Assume that $\mathrm{UB}_{k}$ is the currently known upper bound of the proposed branch and bound algorithm, and for any fixed $\theta_{i}, i=0,1, \ldots, m$, let

$$
\begin{gathered}
\operatorname{PLRB}_{i}\left(\theta_{i}\right)=\sum_{j=1}^{n} \min \left\{\gamma_{i j}\left(\theta_{i}\right) l_{j}, \gamma_{i j}\left(\theta_{i}\right) u_{j}\right\}+\eta_{i}\left(\theta_{i}\right), \\
i=0,1, \ldots, m, \\
\beta_{p}\left(\theta_{0}\right)=\mathrm{UB}_{k}-\operatorname{PLRB}_{0}\left(\theta_{0}\right)+\min \left\{\gamma_{0 p}\left(\theta_{0}\right) l_{p}, \gamma_{0 p}\left(\theta_{0}\right) u_{p}\right\}, \\
p=1, \ldots, n, \\
\lambda_{i p}\left(\theta_{i}\right)=b_{i}-\operatorname{PLRB}_{i}\left(\theta_{i}\right)+\min \left\{\gamma_{i p}\left(\theta_{i}\right) l_{p}, \gamma_{i p}\left(\theta_{i}\right) u_{p}\right\}, \\
p=1, \ldots, n, \quad i=1, \ldots, m .
\end{gathered}
$$

And define $\overline{\bar{Y}}=\left(\overline{\bar{Y}}_{j}\right)_{n \times 1}, \underline{Y}=\left(\underline{Y}_{j}\right)_{n \times 1}, \tilde{Y}=\left(\widetilde{Y}_{j}\right)_{n \times 1}$, and $\widehat{Y}=\left(\widehat{Y}_{j}\right)_{n \times 1}$, where

$$
\begin{aligned}
& \tilde{Y}_{j}= \begin{cases}X_{j}, & j \neq p, j=1, \ldots, n, \\
\left(\frac{\lambda_{i p}\left(\theta_{i}\right)}{\gamma_{i p}\left(\theta_{i}\right)}, u_{p}\right] \bigcap X_{p}, & j=p ;\end{cases} \\
& \widehat{Y}_{j}= \begin{cases}X_{j}, \\
{\left[l_{p}, \frac{\lambda_{i p}\left(\theta_{i}\right)}{\gamma_{i p}\left(\theta_{i}\right)}\right) \bigcap X_{p},} & j=p ;\end{cases} \\
& \overline{\bar{Y}}_{j}= \begin{cases}X_{j}, \\
\left(\frac{\beta_{p}\left(\theta_{0}\right)}{\gamma_{0 p}\left(\theta_{0}\right)}, u_{p}\right] \bigcap X_{p}, & j=p ;\end{cases} \\
& \underline{Y}_{j}= \begin{cases}X_{j}, \\
{\left[l_{p}, \frac{\beta_{p}\left(\theta_{0}\right)}{\gamma_{0 p}\left(\theta_{0}\right)}\right) \bigcap X_{p},} & j=p .\end{cases}
\end{aligned}
$$

Theorem 3. For any subhyperrectangle $X \subseteq X^{0}$, one has the following results.

(i) If $P L R B_{0}\left(\theta_{0}\right)>U B_{k}$, then the subhyperrectangle $X$ does not contain the global optimum point of the problem (NQCQP).

(ii) If $P L R B_{0}\left(\theta_{0}\right) \leq U B_{k}$, then for any $p \in\{1,2, \ldots, n\}$, if $\gamma_{0 p}\left(\theta_{0}\right)>0$, then the subhyperrectangle $\overline{\bar{Y}}=$ $\left(\overline{\bar{Y}}_{j}\right)_{n \times 1}$ does not contain the global optimum point of the problem $(N Q C Q P)$; if $\gamma_{0 p}\left(\theta_{0}\right)<0$, then the subhyperrectangle $\underline{Y}=\left(\underline{Y}_{j}\right)_{n \times 1}$ does not contain the global optimum point of the problem (NQCQP).

(iii) If there exists some $i \in\{1, \ldots, m\}$ such that $P L R B_{i}\left(\theta_{i}\right)>$ $b_{i}$, then the subhyperrectangle $X$ does not contain the global optimum point of the problem (NQCQP).

(iv) If $P L R B_{i}\left(\theta_{i}\right) \leq b_{i}$ for all $i=1, \ldots, m$, then for each $p \in$ $\{1,2, \ldots, n\}$, if $\gamma_{i p}\left(\theta_{i}\right)>0$, then the subhyperrectangle $\widetilde{Y}=\left(\widetilde{Y}_{j}\right)_{n \times 1}$ does not contain the global optimum point 
of the problem (NQCQP); if $\gamma_{i p}\left(\theta_{i}\right)<0$, then the subhyperrectangle $\widehat{Y}=\left(\widehat{Y}_{j}\right)_{n \times 1}$ does not contain the global optimum point of the problem (NQCQP).

Proof. (i) If $\operatorname{PLRB}_{0}\left(\theta_{0}\right)>\mathrm{UB}_{k}$, then for all $x \in X$, by the Theorem 2 we have

$$
\begin{aligned}
G_{0}(x) & \geq G_{0}^{L}\left(x, X, \theta_{0}\right) \\
& \geq \sum_{j=1}^{n} \min \left\{\gamma_{0 j}\left(\theta_{0}\right) l_{j}, \gamma_{0 j}\left(\theta_{0}\right) u_{j}\right\}+\eta_{0}\left(\theta_{0}\right) \\
& =\operatorname{PLRB}_{0}\left(\theta_{0}\right)>\mathrm{UB}_{k} .
\end{aligned}
$$

Hence, the $X$ does not contain the global optimum point of the problem (NQCQP).

(ii) If $\operatorname{PLRB}_{0}\left(\theta_{0}\right) \leq \mathrm{UB}_{k}$, then for any $p \in\{1, \ldots, n\}$, if $\gamma_{0 p}\left(\theta_{0}\right)>0$, for all $x \in \overline{\bar{Y}}$, we have $x_{p}>\beta_{p}\left(\theta_{0}\right) / \gamma_{0 p}\left(\theta_{0}\right)$; that is, $\gamma_{0 p}\left(\theta_{0}\right) x_{p}>\mathrm{UB}_{k}-\operatorname{PLRB}_{0}\left(\theta_{0}\right)+\min \left\{\gamma_{0 p}\left(\theta_{0}\right) l_{p}, \gamma_{0 p}\left(\theta_{0}\right) u_{p}\right\}$. Thus, we have

$$
\begin{aligned}
& G_{0}^{L}\left(x, \overline{\bar{Y}}, \theta_{0}\right) \\
& =\sum_{j=1, j \neq p}^{n} \gamma_{0 j}\left(\theta_{0}\right) x_{j}+\gamma_{0 p}\left(\theta_{0}\right) x_{p}+\eta_{0}\left(\theta_{0}\right) \\
& \geq \sum_{j=1, j \neq p}^{n} \min \left\{\gamma_{0 j}\left(\theta_{0}\right) l_{j}, \gamma_{0 j}\left(\theta_{0}\right) u_{j}\right\}+\eta_{0}\left(\theta_{0}\right)+\gamma_{0 p}\left(\theta_{0}\right) x_{p} \\
& >\sum_{j=1, j \neq p}^{n} \min \left\{\gamma_{0 j}\left(\theta_{0}\right) l_{j}, \gamma_{0 j}\left(\theta_{0}\right) u_{j}\right\}+\eta_{0}\left(\theta_{0}\right) \\
& \quad+\mathrm{UB}_{k}-\mathrm{PLRB}_{0}\left(\theta_{0}\right)+\min \left\{\gamma_{0 p}\left(\theta_{0}\right) l_{p}, \gamma_{0 p}\left(\theta_{0}\right) u_{p}\right\} \\
& =\mathrm{PLRB}_{0}\left(\theta_{0}\right)+\mathrm{UB}_{k}-\mathrm{PLRB}_{0}\left(\theta_{0}\right) \\
& =\mathrm{UB}_{k} .
\end{aligned}
$$

Therefore, by the above inequality and Theorem 2 , we get that

$$
G_{0}(x) \geq G_{0}^{L}\left(x, \overline{\bar{Y}}, \theta_{0}\right)>\mathrm{UB}_{k} .
$$

Hence, the rectangle $\overline{\bar{Y}}$ does not contain the global optimum point of the problem (NQCQP).

Similarly, if $\gamma_{0 p}\left(\theta_{0}\right)<0$, we can prove that the subhyperrectangle $\underline{Y}$ does not contain the global optimum point of the problem (NQCQP).

Using similar proving method as the above, we can draw the conclusions (iii) and (iv).

By Theorem 3, by making use of the above cutting down approach to reject a part of the investigated subhyperrectangle which does not contain the global minimum point of the problem (NQCQP), thus we can enhance the computational speed of the proposed branch and bound algorithm.

\section{Algorithm and Its Convergence}

In this section, based on the former parametric linear programs relaxation problem, we present a novel optimization method for globally solving the problem (NQCQP). There are three fundamental compositions in the presented method: a branching approach, an updating upper bounds approach, and an updating lower bounds approach.

The branching approach iteratively subdivides the investigated hyperrectangle $X^{k}$ into two subhyperrectangles, which produces a more refined partition for computing the global optimum point of the problem (NQCQP). In this paper we select a simple partitioning approach, which is enough to guarantee the global convergence of the presented branch and bound algorithm. For any selected subhyperrectangle $X^{k}=$ $\left[l^{k}, u^{k}\right] \subseteq X^{0}$, the selected partitioning approach is described as follows.

(a) Let $\eta=\arg \max \left\{u_{i}^{k}-l_{i}^{k}: i=1, \ldots, n\right\}$.

(b) Let

$$
\begin{aligned}
& X^{k, 1}=\left\{x \in R^{n} \mid l_{i}^{k} \leq x_{i} \leq u_{i}^{k}, i \neq \eta, l_{\eta}^{k} \leq x_{\eta} \leq \frac{l_{\eta}^{k}+u_{\eta}^{k}}{2}\right\}, \\
& X^{k, 2}=\left\{x \in R^{n} \mid l_{i}^{k} \leq x_{i} \leq u_{i}^{k}, i \neq \eta, \frac{l_{\eta}^{k}+u_{\eta}^{k}}{2} \leq x_{\eta} \leq u_{\eta}^{k}\right\} .
\end{aligned}
$$

By making use of this branching approach, the selected hyperrectangle $X^{k}$ is subdivided into two subhyperrectangles $X^{k, 1}$ and $X^{k, 2}$.

The updating lower bounds approach needs to compute a sequence of parametric linear programs relaxation problems by using the simplex approach. The updating upper bounds approaches need to calculate the objective function value of the feasible point of the problem (NQCQP), where the feasible point can be found by solving the parametric linear programs relaxation problem and probing the feasibility of the midpoint of the investigated subhyperrectangle $X^{k}$, respectively.

4.1. Novel Optimization Algorithm. Let $\operatorname{LB}\left(X^{k}\right)$ and $x^{k}=$ $x\left(X^{k}\right)$ be the optimal value and the optimal solution of the problem (PLPRP) over subhyperrectangle $X^{k}$, respectively. Combining the former parametric linear programs relaxation problem with the cutting down approach in a branch and bound framework, a novel global optimization method for the problem (NQCQP) is described as follows.

\section{Algorithm Steps}

Step 1 (initializing). Initialize the iteration counter $k:=0$, the collection of all active node $\Omega_{0}=\left\{X^{0}\right\}$, the feasible solution set $F=\emptyset$, the convergence error $\epsilon>0$, and the upper bound $\mathrm{UB}_{0}=+\infty$. 
Solve the problem (PLPRP) over the hyperrectangle $X^{0}$ to compute $\mathrm{LB}_{0}:=\operatorname{LB}\left(X^{0}\right)$ and $x^{0}:=x\left(X^{0}\right)$. For all $i=1, \ldots, m$, if $G_{i}\left(x^{0}\right) \leq b_{i}$, then let $F=\left\{x^{0}\right\}$ and $\mathrm{UB}_{0}=G_{0}\left(x^{0}\right)$.

If $\mathrm{UB}_{0}-\mathrm{LB}_{0} \leq \epsilon$, then algorithm terminates with $x^{0}$ as the global optimum point of the problem (NQCQP). Otherwise, go to Step 2.

Step 2 (partitioning hyperrectangle). Utilizing the proposed branching approach, select a branching variable $x_{\eta}$ to partition $X^{k}$ into two new subhyperrectangles, and still let the new collection of partitioned subhyperrectangles by $\bar{X}^{k}$.

Step 3 (cutting down region). For each subhyperrectangle $X \in \bar{X}^{k}$, for any fixed parameter vector $\theta=\left(\theta_{0}, \theta_{1}, \ldots, \theta_{m}\right)$, compute $\operatorname{PLRB}_{i}\left(\theta_{i}\right)(i=0,1, \ldots, m), \beta_{p}\left(\theta_{0}\right)(p=1, \ldots, n)$, and $\lambda_{i p}\left(\theta_{i}\right)(i=1, \ldots, m, p=1, \ldots, n)$.

For each $i \in\{1, \ldots, m\}$, if $\operatorname{PLRB}_{i}\left(\theta_{i}\right)>b_{i}$, let $X=\emptyset$; else

if $\gamma_{i p}\left(\theta_{i}\right)>0$ and $\lambda_{i p}\left(\theta_{i}\right) / \gamma_{i p}\left(\theta_{i}\right)<u_{p}$ for some $p \in$ $\{1, \ldots, n\}$, then let $u_{p}=\lambda_{i p}\left(\theta_{i}\right) / \gamma_{i p}\left(\theta_{i}\right)$.

else if $\gamma_{i p}\left(\theta_{i}\right)<0$ and $\lambda_{i p}\left(\theta_{i}\right) / \gamma_{i p}\left(\theta_{i}\right)>l_{p}$ for some $p \in\{1, \ldots, n\}$, then let $l_{p}=\lambda_{i p}\left(\theta_{i}\right) / \gamma_{i p}\left(\theta_{i}\right)$.

If $\operatorname{PLRB}_{0}\left(\theta_{0}\right)>\mathrm{UB}_{k}$, then let $X=\emptyset$; else

if $\gamma_{0 p}\left(\theta_{0}\right)>0$ and $\beta_{p}\left(\theta_{0}\right) / \gamma_{0 p}\left(\theta_{0}\right)<u_{p}$ for some $p \in$ $\{1, \ldots, n\}$, then let $u_{p}=\beta_{p}\left(\theta_{0}\right) / \gamma_{0 p}\left(\theta_{0}\right)$,

else if $\gamma_{0 p}\left(\theta_{0}\right)<0$ and $\beta_{p}\left(\theta_{0}\right) / \gamma_{0 p}\left(\theta_{0}\right)>l_{p}$ for some $p \in\{1, \ldots, n\}$, then let $l_{p}=\beta_{p}\left(\theta_{0}\right) / \gamma_{0 p}\left(\theta_{0}\right)$.

At last, still let the remaining subhyperrectangle be $X$, and let the remaining partitioned set be $\bar{X}^{k}$.

Step 4 (feasibility fathoming). For each new subhyperrectangle $X \in \bar{X}^{k}$, compute the lower bounds $\operatorname{LB}(X)$ and $x(X)$ by solving the problem (PLPRP) over $X$.

If $\mathrm{LB}(X)>\mathrm{UB}_{k}$, set $\bar{X}^{k}:=\bar{X}^{k} \backslash X$; otherwise, if the midpoint $x^{\text {mid }}$ of $X$ satisfies $G_{i}\left(x^{\text {mid }}\right) \leq b_{i}$ for all $i=1, \ldots, m$, then let $F:=F \cup\left\{x^{\mathrm{mid}}\right\}$, and if $x(X)$ satisfies $G_{i}(x(X)) \leq b_{i}$ for all $i=1, \ldots, m$, then let $F:=F \cup\{x(X)\}$,

Step 5 (renewing bound). Renew the upper bound $\mathrm{UB}_{k}:=$ $\min _{x \in F} G_{0}(x)$. If $F \neq \emptyset$, the best known feasible solution is denoted by $x^{k}:=\arg \min _{x \in F} G_{0}(x)$. Let $\Theta_{k}:=\left(\Theta_{k} \backslash X^{k}\right) \cup \bar{X}^{k}$, and renew the lower bound $\operatorname{LB}_{k}:=\inf _{X \in \Theta_{k}} \operatorname{LB}(X)$.

Step 6 (convergence fathoming). If $\mathrm{UB}_{k}-\mathrm{LB}_{k} \leq \epsilon$, then algorithm stops, and we get that $\mathrm{UB}_{k}$ is the global $\epsilon$-minimum value of the problem (NQCQP), and $x^{k}$ is a global $\epsilon$ optimum point. Otherwise, $k:=k+1$, and select a new subhyperrectangle $X^{k}$ such that $X^{k}=\arg \min _{X \in \Theta_{k}} \operatorname{LB}(X)$, and return to Step 2.

4.2. Global Convergence of the Algorithm. The global convergence of the presented algorithm is described as follows.
Theorem 4. If the proposed algorithm stops finitely at the $k$ th iteration, then when the algorithm stops, $x^{k}$ is the global optimum point of the problem (NQCQP); else it will bring about an infinite sequence $\left\{x^{k}\right\}$ of iteration, such that any accumulation point $x^{*}$ of the sequence $\left\{x^{k}\right\}$ will be the global optimum point of the problem (NQCQP), and the sequence $\left\{U B_{k}\right\}$ is nonincreasing and the sequence $\left\{L B_{k}\right\}$ is nondecreasing; moreover they meet $\lim _{k \rightarrow \infty} U B_{k}=\lim _{k \rightarrow \infty} L B_{k}=$ $v^{*}$, where $v^{*}$ is the global minimum value of the problem (NQCQP).

Proof. (i) If the proposed algorithm stops finitely at the $k$-th iteration, then when it stops, we get that $\mathrm{UB}_{k}=v^{*}=\mathrm{LB}_{k}$. Thus, by the characteristic of the proposed branch and bound algorithm, we get the global optimum point $x^{k}$ of the problem (NQCQP). If the proposed algorithm is infinite, then it must bring about an infinite subhyperrectangle sequence $\left\{X^{k}\right\}$, since the used branching approach is exhaustive, we get that the subhyperrectangle sequence $\left\{X^{k}\right\}$ converges to a point. By the branch and bound characteristic of the algorithm we get that the sequence $\left\{\mathrm{UB}_{k}\right\}$ is nonincreasing, and the sequence $\left\{\mathrm{LB}_{k}\right\}$ is nondecreasing; therefore the sequence $\left\{\mathrm{UB}_{k}-\mathrm{LB}_{k}\right\}$ is a positive and nonincreasing sequence. From Theorem 2 , we know that the sequence $\left\{\mathrm{UB}_{k}-\mathrm{LB}_{k}\right\}$ must be convergent to zero. Also $\mathrm{LB}_{k} \leq v^{*} \leq \mathrm{UB}_{k}$ for each $k$ indicate that $\lim _{k \rightarrow \infty} \mathrm{UB}_{k}=\lim _{k \rightarrow \infty} \mathrm{LB}_{k}=v^{*}$. Since $x^{k}$ is always a feasible solution of the problem (NQCQP) and the upper bound $\mathrm{UB}_{k}=G_{0}\left(x^{k}\right)$, any cluster point $x^{*}$ of the sequence $\left\{x^{k}\right\}$ must be feasible to the problem (NQCQP) with objective function value $v^{*}=G_{0}\left(x^{*}\right)$. Therefore, the conclusion is followed.

\section{Numerical Experiments}

To validate the performance and computational efficiency of the presented optimization method, several common test examples in literatures are put into effect on microcomputer, the algorithm program is coded in $\mathrm{C}++$, the simplex approach is applied to solve a series of parametric linear programs relaxation problems, and the termination tolerance error is set to $\epsilon=10^{-6}$. These test examples are described and their numerical results are listed as follows. In the following Tables 1, 2, and 3, number of algorithm iteration and computational time in seconds are denoted by "Iter" and "Time," respectively.

Example 1 (see [21, 32]). Consider

$$
\begin{array}{ll}
\min & -x_{1}^{2}+x_{1}+x_{2}^{2}-2 x_{2}+x_{1} x_{2} \\
\text { s.t. } & x_{1}+x_{2} \leq 6, \\
& -2 x_{1}^{2}+x_{2}^{2}+2 x_{1}+x_{2} \leq-4, \\
& 1 \leq x_{1}, \quad x_{2} \leq 6 .
\end{array}
$$


TABLE 1: Numerical results for Examples 1-3.

\begin{tabular}{|c|c|c|c|c|c|c|}
\hline Example & Refs. & $\left(\theta_{0}, \ldots, \theta_{m}\right)$ & Optimal solution & Optimal value & Iter & Time (s) \\
\hline \multirow{10}{*}{1} & our1 & $(0,0,0)$ & $(5.0,1.0)$ & -16.000000000 & 3 & 0.00190359 \\
\hline & our2 & $(0,0,1)$ & $(5.0,1.0)$ & -16.000000000 & 2 & 0.000975264 \\
\hline & our3 & $(0,1,1)$ & $(5.0,1.0)$ & -16.000000000 & 2 & 0.000958502 \\
\hline & our4 & $(1,1,1)$ & $(5.0,1.0)$ & -16.000000000 & 3 & 0.00115294 \\
\hline & our5 & $(1,0,0)$ & $(5.0,1.0)$ & -16.000000000 & 4 & 0.0013566 \\
\hline & our6 & $(1,0,1)$ & $(5.0,1.0)$ & -16.000000000 & 3 & 0.00114037 \\
\hline & our7 & $(1,1,0)$ & $(5.0,1.0)$ & -16.000000000 & 4 & 0.00142281 \\
\hline & our8 & $(0,1,0)$ & $(5.0,1.0)$ & -16.000000000 & 3 & 0.00108701 \\
\hline & {$[32]$} & & $(5.0,1.0)$ & -16.0 & 20 & 0.175 \\
\hline & {$[21]$} & & $(5.0,1.0)$ & -16.0 & 5 & 0.00184588 \\
\hline \multirow{6}{*}{2} & our1 & $(0,0)$ & $(2.0,1.666666667)$ & 6.777778018 & 32 & 0.00662822 \\
\hline & our2 & $(0,1)$ & $(2.0,1.666666667)$ & 6.777778233 & 32 & 0.00665057 \\
\hline & our3 & $(1,1)$ & $(2.0,1.666666667)$ & 6.777778517 & 33 & 0.00698972 \\
\hline & our4 & $(1,0)$ & $(2.0,1.666666667)$ & 6.777778743 & 32 & 0.00685227 \\
\hline & {$[19]$} & & $(2.0,1.666666667)$ & 6.777782016 & 40 & 0.032 \\
\hline & {$[22]$} & & $(2.00003,1.66665)$ & 6.7780 & 44 & 0.18 \\
\hline \multirow{11}{*}{3} & our1 & $(0,0,0)$ & $(0.5,0.5)$ & 0.500000000 & 25 & 0.0065648 \\
\hline & our2 & $(0,0,1)$ & $(0.5,0.5)$ & 0.500000000 & 25 & 0.00733278 \\
\hline & our3 & $(0,1,1)$ & $(0.5,0.5)$ & 0.500000600 & 26 & 0.00686791 \\
\hline & our4 & $(1,1,1)$ & $(0.5,0.5)$ & 0.500000600 & 26 & 0.00691568 \\
\hline & our5 & $(1,0,0)$ & $(0.5,0.5)$ & 0.500000000 & 25 & 0.00735261 \\
\hline & our6 & $(1,0,1)$ & $(0.5,0.5)$ & 0.500000000 & 25 & 0.00736742 \\
\hline & our7 & $(1,1,0)$ & $(0.5,0.5)$ & 0.500000600 & 26 & 0.00769232 \\
\hline & our8 & $(0,1,0)$ & $(0.5,0.5)$ & 0.500000600 & 26 & 0.00921374 \\
\hline & {$[19]$} & & $(0.5,0.5)$ & 0.500004627 & 34 & 0.056 \\
\hline & {$[21]$} & & $(0.5,0.5)$ & 0.500000442 & 37 & 0.0192625 \\
\hline & {$[22]$} & & $(0.5,0.5)$ & 0.5 & 91 & 0.85 \\
\hline
\end{tabular}

Example 2 (see $[19,21,22])$. Consider

$$
\begin{array}{ll}
\min & x_{1}^{2}+x_{2}^{2} \\
\text { s.t. } & -0.3 x_{1} x_{2} \leq-1, \\
& 2 \leq x_{1} \leq 5, \quad 1 \leq x_{2} \leq 3 .
\end{array}
$$

Example 3 (see [19, 21, 22]). Consider

$$
\begin{array}{ll}
\min & x_{1} \\
\text { s.t. } & 4 x_{2}-4 x_{1}^{2} \leq 1, \\
& x_{1}+x_{2} \geq 1, \\
& 0.01 \leq x_{1}, \quad x_{2} \leq 15 .
\end{array}
$$

Example 4 (see [21, 23]). Consider

$$
\begin{array}{ll}
\min & x_{1} x_{2}-2 x_{1}+x_{2}+1 \\
\text { s.t. } & 8 x_{2}^{2}-6 x_{1}-16 x_{2} \leq-11 \\
& -x_{2}^{2}+3 x_{1}+2 x_{2} \leq 7 \\
& 1 \leq x_{1} \leq 2.5, \quad 1 \leq x_{2} \leq 2.225 .
\end{array}
$$

Example 5 (see $[17,21])$. Consider

$$
\begin{array}{ll}
\min & 6 x_{1}^{2}+4 x_{2}^{2}+5 x_{1} x_{2} \\
\text { s.t. } & -6 x_{1} x_{2} \leq-48, \quad 0 \leq x_{1}, x_{2} \leq 10 .
\end{array}
$$

Example 6 (see [21, 25]). Consider

$$
\begin{array}{ll}
\min & -4 x_{2}+\left(x_{1}-1\right)^{2}+x_{2}^{2}-10 x_{3}^{2} \\
\min & 6 x_{1}^{2}+4 x_{2}^{2}+5 x_{1} x_{2} \\
& \left(x_{1}-2\right)^{2}+x_{2}^{2}+x_{3}^{2} \leq 2, \\
& 2-\sqrt{2} \leq x_{1} \leq \sqrt{2}, \quad 0 \leq x_{2}, \quad x_{3} \leq \sqrt{2} .
\end{array}
$$

Example 7 (see [33, 34]). Consider

$$
\begin{gathered}
\min x_{1}+x_{2}+x_{3} \\
\text { s.t. } \quad 833.33252 x^{-1} x_{4} x^{-6}+100.0 x_{6}^{-1} \\
-83333.333 x_{1}^{-1} x_{6}^{-1} \leq 1 \\
1250.0 x_{2}^{-1} x_{5} x_{7}^{-1}+1.0 x_{4} x_{7}^{-1} \\
-1250.0 x_{2}^{-1} x_{4} x_{7}^{-1} \leq 1
\end{gathered}
$$


TABLE 2: Numerical results for Examples 4-6.

\begin{tabular}{|c|c|c|c|c|c|c|}
\hline Example & Refs. & $\left(\theta_{0}, \ldots, \theta_{m}\right)$ & Optimal solution & Optimal value & Iter & Time (s) \\
\hline \multirow{10}{*}{4} & our1 & $(0,0,0)$ & $(2.0,1.0)$ & -1.000000 & 0 & 0.00071504 \\
\hline & our2 & $(0,0,1)$ & $(2.0,1.0)$ & -1.000000000 & 1 & 0.00165866 \\
\hline & our3 & $(0,1,1)$ & $(2.0,1.0)$ & -1.000000000 & 2 & 0.00169589 \\
\hline & our4 & $(1,1,1)$ & $(2.0,1.0)$ & -1.000000000 & 2 & 0.00156529 \\
\hline & our5 & $(1,0,0)$ & $(2.0,1.0)$ & -1.000000000 & 2 & 0.00153087 \\
\hline & our6 & $(1,0,1)$ & $(2.0,1.0)$ & -1.000000000 & 2 & 0.00159881 \\
\hline & our7 & $(1,1,0)$ & $(2.0,1.0)$ & -1.000000000 & 2 & 0.00158519 \\
\hline & our8 & $(0,1,0)$ & $(2.0,1.0)$ & -1.000000 & 0 & 0.00066607 \\
\hline & {$[21]$} & & $(2.0,1.0)$ & -0.999999410 & 21 & 0.00849446 \\
\hline & {$[23]$} & & $(2.0,1.0)$ & -1.0 & 24 & 0.0116923 \\
\hline \multirow{6}{*}{5} & ourl & $(0,0)$ & $(2.555409888,3.130613160)$ & 118.383672050 & 49 & 0.0271463 \\
\hline & our2 & $(0,1)$ & $(2.555676683,3.130286407)$ & 118.383672115 & 51 & 0.0287108 \\
\hline & our3 & $(1,1)$ & $(2.555800904,3.130134268)$ & 118.383672231 & 61 & 0.0350215 \\
\hline & our4 & $(1,0)$ & $(2.555775920,3.130164866)$ & 118.383671937 & 55 & 0.030462 \\
\hline & {$[17]$} & & $(2.555779370,3.130164640)$ & 118.383756475 & 210 & 0.78 \\
\hline & {$[21]$} & & $(2.555745855,3.130201688)$ & 118.383671904 & 59 & 0.0385038 \\
\hline \multirow{10}{*}{6} & ourl & $(0,0,0)$ & $(1.0,0.181818247,0.983332154)$ & -11.363635387 & 141 & 0.110562 \\
\hline & our2 & $(0,0,1)$ & $(1.0,0.181818217,0.983332160)$ & -11.363636364 & 240 & 0.198587 \\
\hline & our3 & $(0,1,1)$ & $(1.0,0.181818196,0.983332163)$ & -11.363635785 & 260 & 0.213692 \\
\hline & our4 & $(1,1,1)$ & $(1.0,0.181818133,0.983332175)$ & -11.363635682 & 229 & 0.18844 \\
\hline & our5 & $(1,0,0)$ & $(1.0,0.181783067,0.983338664)$ & -11.363635889 & 80 & 0.0669022 \\
\hline & our6 & $(1,0,1)$ & $(1.0,0.181818133,0.983332175)$ & -11.363635715 & 213 & 0.176459 \\
\hline & our7 & $(1,1,0)$ & $(1.0,0.181818301,0.983332144)$ & -11.363635516 & 149 & 0.131391 \\
\hline & our8 & $(0,1,0)$ & $(1.0,0.181818133,0.983332175)$ & -11.363636364 & 133 & 0.105004 \\
\hline & {$[21]$} & & $(1.0,0.181818470,0.983332113)$ & -11.363636364 & 420 & 0.284541 \\
\hline & {$[25]$} & & $(0.998712,0.196213,0.979216)$ & -10.35 & 1648 & 0.3438 \\
\hline
\end{tabular}

TABLE 3: Numerical comparisons with Refs. [16, 21] for Example 9.

\begin{tabular}{lccccc}
\hline Refs. & Number of variable & $n=5$ & $n=10$ & $n=20$ & $n=30$ \\
\hline \multirow{2}{*}{ Ours } & Computational time (s) & 0.0124692 & 0.215709 & 3.69194 & 27.4522 \\
& Iteration Number & 12 & 31 & 204 \\
\hline \multirow{2}{*}{ Ref. [16] } & Computational time (s) & 10.11 & 21.86 & 47.00 & 106.33 \\
& Iteration Number & 141 & 283 & 651 \\
\hline \multirow{2}{*}{ Ref. [21] } & Computational time (s) & 0.0181791 & 0.302157 & 6.01095 \\
& Iteration Number & 12 & 32 & 865 \\
\hline
\end{tabular}

$$
\begin{gathered}
1250000.0 x_{3}^{-1} x_{8}^{-1}+1.0 x_{5} x_{8}^{-1} \\
-2500.0 x_{3}^{-1} x_{5} x_{8}^{-1} \leq 1, \\
0.0025 x_{4}+0.0025 x_{6} \leq 1, \\
-0.0025 x_{4}+0.0025 x_{5} \\
+0.0025 x_{7} \leq 1, \\
0.001 x_{8}-0.001 x_{5} \leq 1, \\
(100,1000,1000,10,10,10,10,10) \\
\leq\left(x_{1}, x_{2}, x_{3}, x_{4}, x_{5}, x_{6}, x_{7}, x_{8}\right),
\end{gathered}
$$

$$
\begin{array}{r}
\left(x_{1}, x_{2}, x_{3}, x_{4}, x_{5}, x_{6}, x_{7}, x_{8}\right) \\
\leq(10000,10000,10000,1000, \\
1000,1000,1000,1000) .
\end{array}
$$

This problem is from the heat exchanger design problem; solve this problem by the proposed method, with the given convergence error $\epsilon=10^{-6}$ and parameter vector $\theta_{i}=0$, $i=0,1, \ldots, 6$, and a global $\epsilon$-optimal solution

$$
\left(x_{1}, \ldots, x_{8}\right)^{T}=(579.307,1359.971,5109.971,182.018,
$$

$295.601,217.982,286.417,395.6)$ 
is found after 18377 iterations with objective function value 7049.248020529 , and computational time is $11.475 \mathrm{~s}$.

Using the proposed approach in Floudas et al. [33], a global optimal solution, under the given convergence tolerance $\epsilon=10^{-4}$,

$$
\left(x_{1}, \ldots, x_{8}\right)^{T}=(579.31,1359.97,5109.97,182.01 \text {, }
$$

$$
295.60,217.98,286.42,395.6)
$$

is found with global optimal value 7049.25000.

Using the proposed approach in Lin and Tsai [34], under the convergence tolerance $\epsilon=10^{-6}$, a global optimal solution

$$
\left(x_{1}, \ldots, x_{8}\right)^{T}=(578.973,1359.573,5110.701,181.99 \text {, }
$$

$$
295.572,218.01,286.418,395.572)
$$

is found with global optimization value 7049.24692.

Example 8 (see [24]). Consider

$$
\begin{gathered}
\min \quad 5.3578 x_{3}^{2}+0.8357 x_{1} x_{5}+37.2392 x_{1} \\
\text { s.t. } \quad 0.00002584 x_{3} x_{5}-0.00006663 x_{2} x_{5} \\
-0.0000734 x_{1} x_{4} \leq 1, \\
0.000853007 x_{2} x_{5}+0.00009395 x_{1} x_{4} \\
-0.00033085 x_{3} x_{5} \leq 1, \\
1330.3294 x_{2}^{-1} x_{5}^{-1}-0.42 x_{1} x_{5}^{-1} \\
-0.30586 x_{2}^{-1} x_{3}^{2} x_{5}^{-1} \leq 1, \\
0.00024186 x_{2} x_{5}+0.00010159 x_{1} x_{2} \\
+0.00007379 x_{3}^{2} \leq 1, \\
2275.1327 x_{3}^{-1} x_{5}^{-1}-0.2668 x_{1} x_{5}^{-1} \\
\quad 0.40584 x_{4} x_{5}^{-1} \leq 1, \\
0.00029955 x_{3} x_{5}+0.00007992 x_{1} x_{3} \\
+0.00012157 x_{3} x_{4} \leq 1, \\
78.0 \leq x_{1} \leq 102.0, \quad 33.0 \leq x_{2} \leq 45.0, \\
27.0 \leq x_{3} \leq 45.0, \\
27.0 \leq x_{4} \leq 45.0, \quad 27.0 \leq x_{5} \leq 45.0 .
\end{gathered}
$$

This test problem has a relative high degree of difficulty, and it contains both negative and positive terms. By using the proposed algorithm in this paper, initializing the parameter $\theta_{i}=1, i=0,1, \ldots, 6$, with the given convergence error $\epsilon=$ $10^{-6}$, the global $\epsilon$-optimal solution

$$
\left(x_{1}, x_{2}, \ldots, x_{5}\right)^{T}=(78.0,33.739811424,30.594417922,
$$

$44.991210938,35.288085938)$

can be obtained after the 51 iterations.
But using the proposed algorithm in [24], with the given convergence error $\epsilon=10^{-6}$, the global $\epsilon$-optimal solution

$$
\left(x_{1}, x_{2}, \ldots, x_{5}\right)^{T}=(78.0,32.99999946,29.995510365 \text {, }
$$

$$
44.999998630,36.77517397)
$$

can be obtained after the 523 iterations.

Example 9 (see $[16,21])$. Consider

$$
\begin{array}{ll}
\min & -\sum_{i=1}^{n} x_{i}^{2} \\
\text { s.t. } & \sum_{i=1}^{j} x_{i} \leq j, \quad j \in\{1,2, \ldots, n\}, \\
& x_{i} \geq 0, \quad i \in\{1,2, \ldots, n\} .
\end{array}
$$

Using the proposed algorithm in this paper, initializing the parameter $\theta_{i}=0, i=0,1, \ldots, n$, the numerical results are compared with those in $[16,21]$ and are illustrated in Table 3.

From the numerical results for Examples 1-9, our algorithm is competitive.

\section{Concluding Remarks}

In this paper, a novel optimization method based on the parametric linear programs relaxation problem is proposed for globally solving the NQCQP problem. The parametric linear programs relaxation problem is constructed by underestimating each quadratic function with a parametric linear function. By making use of the currently known upper bound and the parametric linear programs relaxation of the problem (NQCQP), a cutting down approach is constructed and used to enhance the computational speed of the branch and bound algorithm. The algorithm is convergent to the global optimum point by subdividing the initial hyperrectangle and solving sequences of parametric linear programs relaxation problems. Numerical experimental results are reported to demonstrate that the presented method can be employed to effectively solve the problem (NQCQP).

\section{Conflict of Interests}

The authors declare that there is no conflict of interests regarding the publication of this paper.

\section{Acknowledgments}

This research work is supported by the National Natural Science Foundation of China under Grant (11171094) and the Science and Technology Key Project of Education Department of Henan Province (14A110024). 


\section{References}

[1] V. Visweswaran and C. A. Floudas, "A global optimization algorithm (GOP) for certain classes of nonconvex NLPs II: applications of theory and test problems," Computers and Chemical Engineering, vol. 14, pp. 1417-1434, 1990.

[2] D. P. Rutenberg and T. L. Shaftel, "Product design: subassemblies for multiple markets," Managment Science, vol. 18, no. 4, pp. B220-B231, 1971.

[3] E. V. Demands and C. S. Tang, "Linear control of a Markov production system," Operations Research, vol. 40, pp. 259-278, 1992.

[4] E. Phan-huy-Hao, "Quadratically constrained quadratic programming: some applications and a method for solution," Zeitschrift für Operations Research A, vol. 26, no. 3, pp. 105-119, 1982.

[5] A. Weintraub and J. Vera, "A cutting plane approach for chance constrained linear programs," Operations Research, vol. 39, no. 5, pp. 776-785, 1991.

[6] T. Q. Phong, P. D. Tao, and L. T. H. An, "A method for solving d.c. programming problems: application to fuel mixture nonconvex optimization problem," Journal of Global Optimization, vol. 6, no. 1, pp. 87-105, 1995.

[7] F. A. Al-Khayyal, C. Larsen, and T. van Voorhis, "A relaxation method for nonconvex quadratically constrained quadratic programs," Journal of Global Optimization, vol. 6, no. 3, pp. 215230, 1995.

[8] F. Al-Khayyal and T. van Voorhis, "Accelerating convergence of branch and bound algorithms for quadratically constrained optimization problems," in State of the Art in Global Optimization: Computational Methods and Applications, C. A. Floudas, Ed., vol. 7, Kluwer Academic, London, UK, 1996.

[9] J. A. Filar and T. A. Schultz, "Bilinear programming and structured stochastic games," Journal of Optimization Theory and Applications, vol. 53, no. 1, pp. 85-104, 1987.

[10] H. D. Sherali and C. H. Tuncbilek, "A reformulationconvexification approach for solving nonconvex quadratic programming problems," Journal of Global Optimization, vol. 7, no. 1, pp. 1-31, 1995.

[11] T. Van Voorhis, "A global optimization algorithm using Lagrangian underestimates and the interval Newton method," Journal of Global Optimization, vol. 24, no. 3, pp. 349-370, 2002.

[12] J. Linderoth, "A simplicial branch-and-bound algorithm for solving quadratically constrained quadratic programs," Mathematical Programming B, vol. 103, no. 2, pp. 251-282, 2005.

[13] S. Burer and D. Vandenbussche, "A finite branch-and-bound algorithm for nonconvex quadratic programming via semidefinite relaxations," Mathematical Programming A, vol. 113, no. 2, pp. 259-282, 2008.

[14] X. J. Zheng, X. L. Sun, and D. Li, "Convex relaxations for nonconvex quadratically constrained quadratic programming: matrix cone decomposition and polyhedral approximation," Mathematical Programming B, vol. 129, no. 2, pp. 301-329, 2011.

[15] N. V. Thoai, "Duality bound method for the general quadratic programming problem with quadratic constraints," Journal of Optimization Theory and Applications, vol. 107, no. 2, pp. 331354, 2000.

[16] Y. Gao, H. Xue, and P. Shen, "A new rectangle branch-andreduce approach for solving nonconvex quadratic programming problems," Applied Mathematics and Computation, vol. 168, no. 2, pp. 1409-1418, 2005.
[17] Y. Gao, Y. Shang, and L. Zhang, "A branch and reduce approach for solving nonconvex quadratic programming problems with quadratic constraints," OR Transactions, vol. 9, no. 2, pp. 9-20, 2005.

[18] P. Shen, Y. Duan, and Y. Ma, "A robust solution approach for nonconvex quadratic programs with additional multiplicative constraints," Applied Mathematics and Computation, vol. 201, no. 1-2, pp. 514-526, 2008.

[19] P. P. Shen and L. M. Liu, "A global optimization approach to quadratic programming problems with nonconvex quadratic constraints," Chinese Journal of Engineering Mathematics, vol. 25, no. 5, pp. 923-926, 2008.

[20] S.-J. Qu, K.-C. Zhang, and Y. Ji, "A global optimization algorithm using parametric linearization relaxation," Applied Mathematics and Computation, vol. 186, no. 1, pp. 763-771, 2007.

[21] H. Jiao and Y. Chen, "A global optimization algorithm for generalized quadratic programming," Journal of Applied Mathematics, vol. 2013, Article ID 215312, 9 pages, 2013.

[22] Y. Wang and Z. Liang, "A deterministic global optimization algorithm for generalized geometric programming," Applied Mathematics and Computation, vol. 168, no. 1, pp. 722-737, 2005.

[23] P. Shen and H. Jiao, "A new rectangle branch-and-pruning approach for generalized geometric programming," Applied Mathematics and Computation, vol. 183, no. 2, pp. 1027-1038, 2006.

[24] P. Shen, "Linearization method of global optimization for generalized geometric programming," Applied Mathematics and Computation, vol. 162, no. 1, pp. 353-370, 2005.

[25] P. Shen and X. Li, "Branch-reduction-bound algorithm for generalized geometric programming," Journal of Global Optimization, vol. 56, no. 3, pp. 1123-1142, 2013.

[26] X. P. Hou, P. P. Shen, and Y. Q. Chen, "A global optimization algorithm for signomial geometric programming problem," Abstract and Applied Analysis. In press.

[27] J.-F. Tsai, M.-H. Lin, and Y.-C. Hu, "On generalized geometric programming problems with non-positive variables," European Journal of Operational Research, vol. 178, no. 1, pp. 10-19, 2007.

[28] A. Nazemi and E. Sharifi, "Solving a class of geometric programming problems by an efficient dynamic model," Communications in Nonlinear Science and Numerical Simulation, vol. 18, no. 3, pp. 692-709, 2013.

[29] P.-P. Shen and X.-D. Bai, "Global optimization for generalized geometric programming problems with discrete variables," Optimization, vol. 62, no. 7, pp. 895-917, 2013.

[30] K. Lange and H. Zhou, "MM algorithms for geometric and signomial programming," Mathematical Programming, vol. 143, no. 1-2, pp. 339-356, 2014.

[31] C. A. Floudas and C. E. Gounaris, "A review of recent advances in global optimization," Journal of Global Optimization, vol. 45, no. 1, pp. 3-38, 2009.

[32] P. P. Shen and L. M. Liu, "A linearization method for the global optimal solution of a quadratic programming problem with nonconvex quadratic constraints," Numerical Mathematics, vol. 30, no. 3, pp. 261-267, 2008.

[33] C. A. Floudas, P. M. Pardalos, C. S. Adjiman et al., Handbook of Test Problems in Local and Global Optimization, vol. 33, Kluwer Academic, Boston, Mass, USA, 1999.

[34] M.-H. Lin and J.-F. Tsai, "Range reduction techniques for improving computational efficiency in global optimization of signomial geometric programming problems," European Journal of Operational Research, vol. 216, no. 1, pp. 17-25, 2012. 


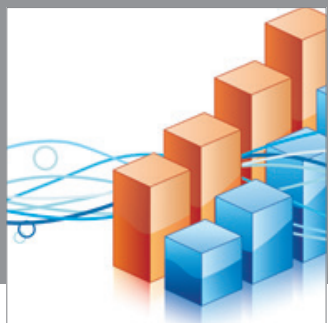

Advances in

Operations Research

mansans

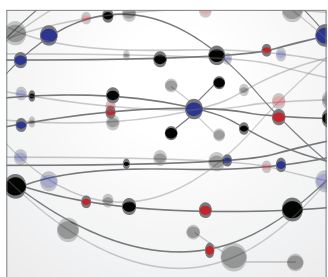

The Scientific World Journal
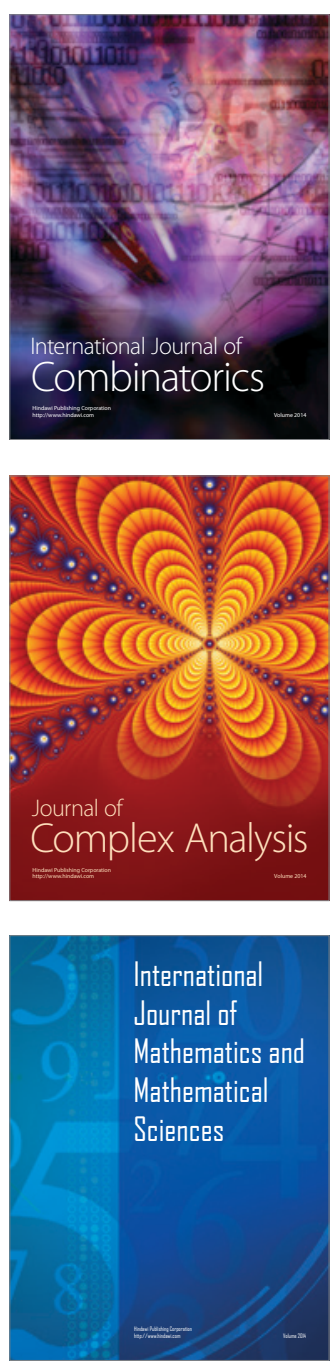
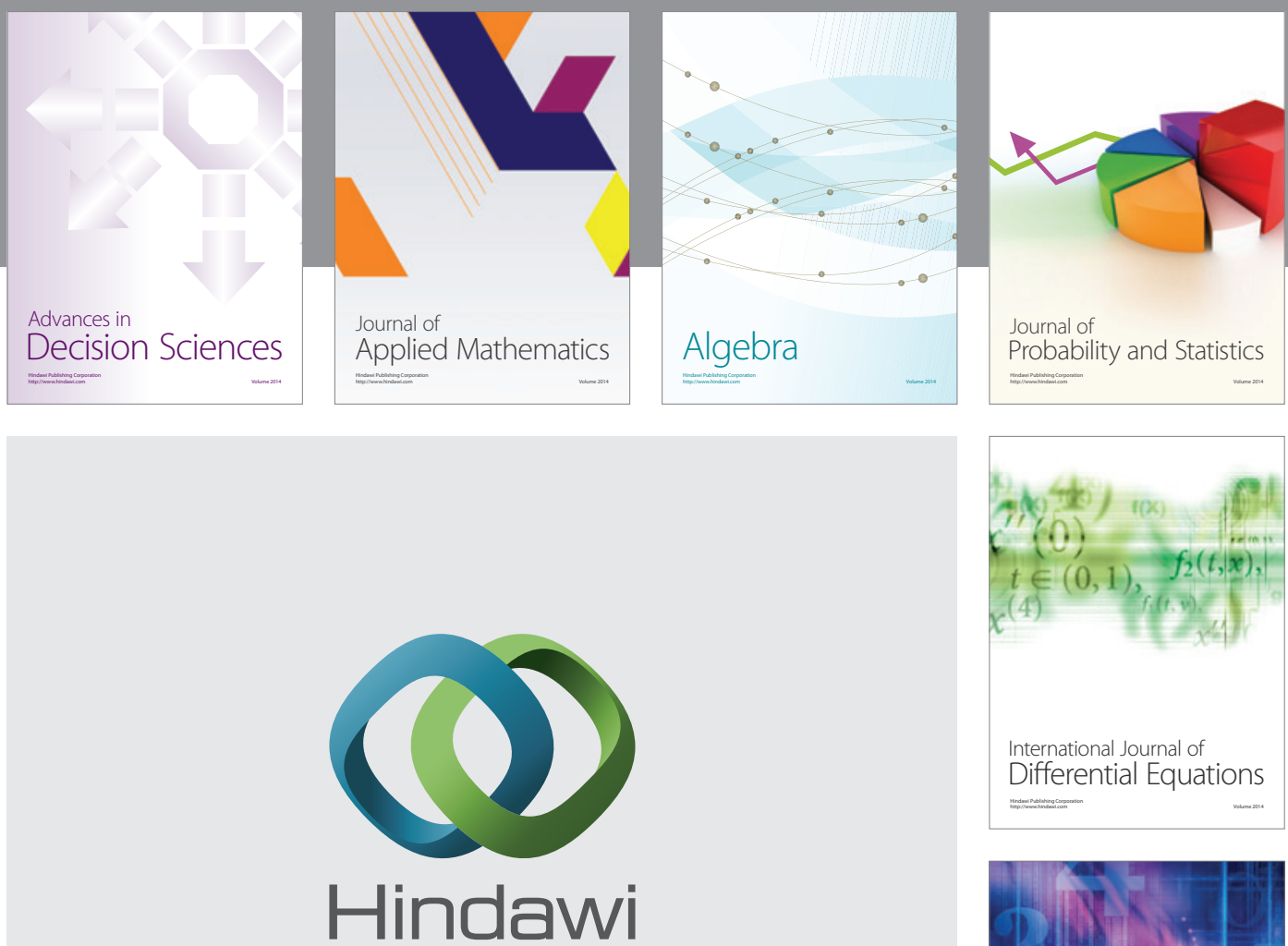

Submit your manuscripts at http://www.hindawi.com
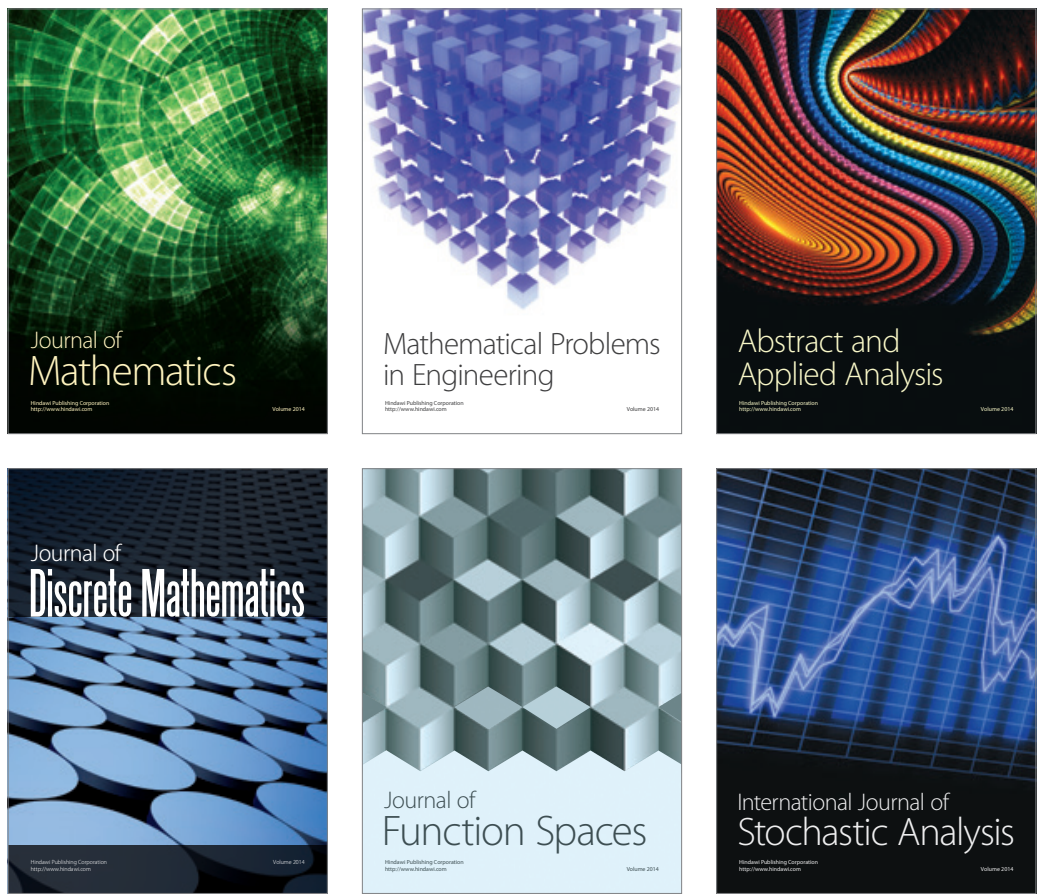

Journal of

Function Spaces

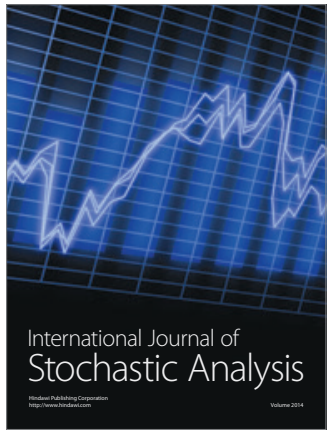

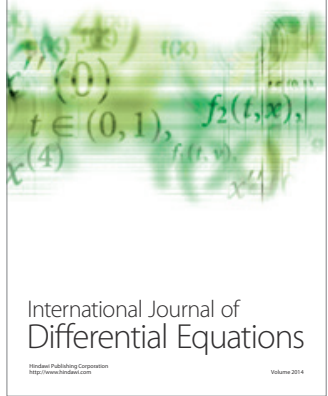
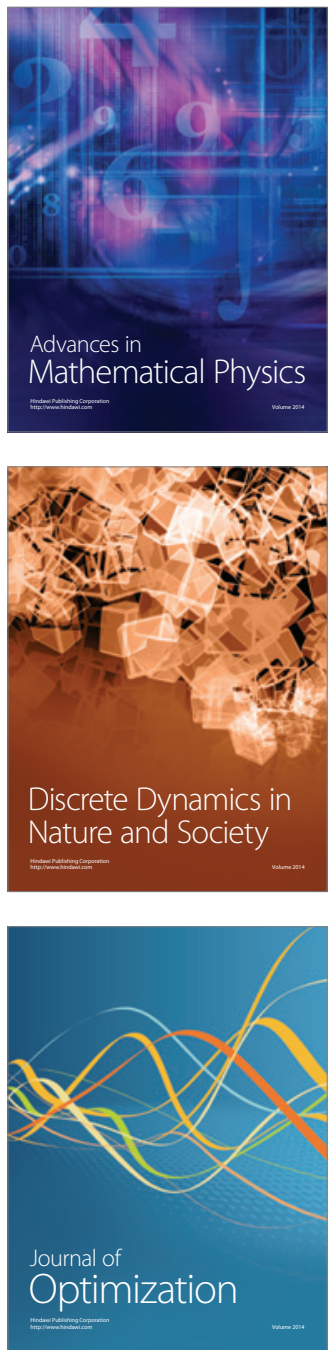